\title{
Localization and Some Properties of Certain Types of Modules
}

\author{
Adil Kadir Jabbar ${ }^{1}$ and Rasti Raheem Mohammad Amin ${ }^{2}$ \\ ${ }^{1}$ Department of Mathematics, College of Science, University of Sulaimani, Sulaimani, Iraq \\ ${ }^{2}$ Department of Mathematics, College of Education, University of Garmian, Garmian, Iraq \\ adilkj@gmail.com or adil.jabbar@univsul.edu.iq and ${ }^{2}$ rasti.raheem90@gmail.com
}

\begin{abstract}
In this paper Artinian and locally prime modules are studied and some characterizations of locally prime modules are given. Some conditions are given under which locally prime modules are almost prime modules and a nonzero module is a locally prime module. Some properties of Artinian and locally Artinian modules are given. Also, strongly reduced modules, primally reduced modules, radically reduced modules and some other types are studied and investigated and some properties of these types of modules are proved. In addition, some relations that concerning these types of modules are established and some characterizations of them are given.
\end{abstract}

Keywords: Artinian and locally Artinian modules, locally prime modules, strongly reduced modules, primally reduced modules and radically reduced modules.

\section{Introduction}

Let $M$ be an $R$-module. A nonempty subset $S$ of $R$ is called a multiplicative system in $R$, if $0 \notin S$ and $a, b \in S$ implies that $a b \in S$ [10]. Let $M$ be an $R$ - module and $N$ is a submodule of $M$, the annihilator of $N$ is defined as $\operatorname{Ann}(N)=\{r \in M: r N=0\}$ [11]. As especial case, we have, $\operatorname{Ann}(M)=\{r \in R: r M=0\}$. Let $N$ be a submodule of an $R$-module $M$, then we define $(N: M)$ as $(N: M)=\{r \in R: r M \subseteq N\} \quad$ [12]. In particular, $(0: M)=\{r \in R: r M=0\}=\operatorname{Ann}(M)$. Let $N$ be a proper submodule of an $R$-module $M$, then $N$ is called a prime submodule of $M$, if $r \in R$ and $x \in M$ such that $r x \in N$, then $x \in N$ or $r M \subseteq N$ [4]. Let $N$ be a proper submodule of an $R$-module $M$, then $N$ is called a semiprime submodule of $M$, if $r \in R$ and $x \in M$ such that $r^{2} x \in N$, then $r x \in N$ and $M$ is called a semiprime module if the zero submodule of $M$ is a semiprime submodule [4]. An $R-$ module $M$ is called a prime module if the zero submodule of $M$ is a prime submodule of $M$ [2]. An $R$-module $M$ is called an almost prime module if each nonzero proper direct summand of $M$ is a prime submodule of $M$ [4]. An $R$-module $M$ is called a fully prime module if every proper submodule of $M$ is prime and it is called an almost fully prime module, if every non zero proper submodule of $M$ is prime [4]. An $R$-module $M$ is called a fully semiprime module if each proper submodule of $M$ is semiprime and 
it is called almost fully semiprime if each nonzero proper submodule of $M$ is semiprime [4]. An $R$-module $M$ is called a locally prime module if $M_{P}$ is a prime module for each maximal ideal $P$ of $R$ [8]. An $R$-module $M$ is called an Artinian module if it satisfies the descending chain condition on submodules, equivalently, if there exists a positive integer $k$ such that $N_{k}=N_{k+n}$, for all $n \in Z^{+}$and it is called a locally Artinian module if $M_{P}$ is an Artinian $R_{P}$ - module for each maximal ideal $P$ of $R$ [8]. The prime spectrum of an $R$-module $M$ is denoted by $\operatorname{Spec}(M)$ and defined as $\operatorname{Spec}(M)=\{N: N$ is a prime submodule of $M\}$ [3]. If $N$ is a submodule of $M$, then $S_{M}(N)=\{r \in R: r m \in N$, for some $m \notin N\}$ [1]. A proper submodule $N$ of $M$ is called a primal submodule if $S_{M}(N)$ forms an ideal of $R$, this ideal is a proper ideal of $R$ [1]. A proper submodule $N$ of an $R$-module $M$ is said to be a weakly prime submodule, if whenever $0 \neq r m \in N$, for $r \in R, m \in M$, then $m \in N$ or $r M \subseteq N$ [2]. An $R$ - module $M$ is called a faithful module if $\operatorname{Ann}(M)=0((0: M)=0)$ [8]. An $R$-module $M$ is called a cyclic $R$ - module if $M=R x$, for some $x \in M$ [8]. Let $M$ be an $R$-module. The primal spectrum of $M$ is denoted by $p \operatorname{Spec}(M)$, and is defind as $p \operatorname{Spec}(M)=\{N: N$ is a primal submodule of $M$ \} and we say that $M$ is a primally reduced $R$ - module if $\cap p \operatorname{Spec}(M)=0$ [5]. An $R$ - module $M$ is called a reduced module if $\cap \operatorname{Spec}(M)=0$ and it is called locally reduced if $M_{P}$ is reduced [6]. Let $M$ be an $R$ - module. The Jacobson spectrum of $M$, denoted by $\operatorname{ipSpec}(M)=\left\{N \in \operatorname{pSpec}(M): S_{M}(N) \subseteq \operatorname{Rad}_{j}(R)\right\}$, where $\operatorname{Rad}_{j}(R)$ is the Jacobson radical of $R$ and we say that $M$ is radically reduced if $\cap j p \operatorname{Spec}(M)=0$ [5]. Let $M$ be an $R$ - module and $P$ a maximal ideal of $R$, we define $\operatorname{Spec}_{p}(M)=\left\{N: N \in \operatorname{Spec}(M)\right.$ and $\left.S_{M}(N) \subseteq P\right\}$ and we say that $M$ is a strongly reduced $R$-module if $\cap \operatorname{Spec}_{p}(M)=0$ [8]. A proper submodule $N$ of $M$ is called a maximal submodule if it is not properly contained in any proper submodule of $M$ and the Jacobson radical of $R$, denoted by $\operatorname{Rad}_{j}(R)$ (or $J(R)$ ), is defined to be the intersection of all the maximal ideal of $R$ [5]. Let $M$ be an $R$-module. A submodule $A$ of $M$ is called an essential submodule of $M$ (or $M$ is an essential extension of $A$ ), written $A \leq_{e} M$, if $B$ is any nonzero submodule of $M$, then $A \cap B \neq\{0\}$, that means every non zero submodule of $M$ must contain at least a non zero element of $A$. Let $M$ be an $R$-module and $A, B$ are submodules of $M$, then the set $\{A, B\}$ is called independent if $A \cap B=\{0\}$. Let $M$ be an $R$-module and $A$ a submodule of $M$. A submodule $B$ of $M$ is said to be relative complement for $A$ if $A \cap B=\{0\}$ and $B$ is maximal with respect to the property $A \cap B=\{0\}$ (that is, $B$ is not contained properly in any other submodule $B$ with the property $\cap \hat{B}=\{0\}$ ). Let $M$ be an $R$-module. A(proper) submodule $A$ of $M$ is called a closed submodule of $M$, written $A \leq_{C} M$, if $A$ has no proper essential extension in $M$ and if the

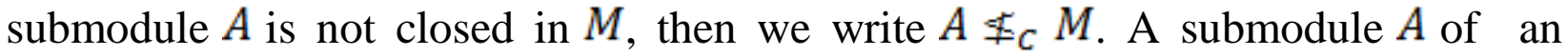


$R$-module $M$ is called small ( or superfluous), in symbols $A \ll M$, if $B$ is any submodule of $M$ such that $A+B=M$, then $B=M$ (equivalently, if $M$ is the only submodule of $M$ such that $A+M=M)$. Let $M$ be an $R$-module. $M$ is called a multiplication module if for each submodule $N$ of $M$, there exists an ideal $I$ of $R$ such that $N=I M$ [8]. Let $M$ be an $R$-module. $M$ is called a weak multiplication module if for every prime submodule $N$ of $M$ we have $N=(N: M) M$ [8]. Let $R$ be a commutative ring with identity. Then $R$ it is called a local ring if it has a unique maximal ideal.

\section{Artinian and Locally Prime Modules}

This section is devoted to study Artinian and locally prime modules. Some characterizations of locally prime modules are given and some conditions are given under which locally prime modules are almost prime modules and also we give a condition which makes a nonzero module as a locally prime module and some properties of Artinian and locally Artinian modules are given.

In the first result we give some characterizations of locally prime modules. Theorem 2.1. Let $M$ be an $R$-module. If $P$ is a maximal ideal of $R$ such that $S_{M}(0) \subseteq P$, then the following conditions are equivalent:

(1) $M$ is a locally prime module.

(2) Each proper direct summand of $M$ is a prime submodule (that is, each nonzero summand becomes a prime module by itself).

(3) All nonzero cyclic submodules of $M$ are isomorphic.

(4) For all $0 \neq m \in M$, we have $\operatorname{Ann}(m)=\operatorname{Ann}(M)$.

Proof. Since, $M$ is locally prime, so that $M_{P}$ is a prime module and as $S_{M}(0) \subseteq P$, the results will follow directly by [9, Theorem 2.10].

The following theorem proves that under certain conditions locally prime modules become almost prime modules.

Theorem 2.2. If $M$ is a locally prime module with $S_{M}(0) \subseteq P$ and $P$ is a maximal ideal of $R$, then $M$ is an almost prime $R$-module.

Proof. Since, $M$ is a locally prime module that means $M_{P}$ is a prime module, then by [9, Theorem 2.12], we get $M$ is an almost prime $R$-module.

In the following result we give some conditions under which a nonzero module is locally prime.

Theorem 2.3. If $M$ is a nonzero $R$ - module such that $S_{M}(0) \subseteq J(R)$ and $S_{M}(0) \subseteq(0: M)$, then $M$ is a locally prime module.

Proof. Let $P$ be any maximal ideal of $R$, then $S_{M}(0) \subseteq J(R) \subseteq P$, so by [7, Theorem 2.12], we get $\{0\}$ is a prime submodule of $M$ and by [7, Proposition 2.17], we have $\{0\}_{P}$ is a proper submodule of $M_{P}$. To show $\{0\}_{P}$ is a prime submodule of $M_{P}$. Let $\quad \frac{r}{p} \frac{a}{q} \in\{0\}_{P}$, where $r \in R, a \in M, p, q \notin P$ and let $\frac{a}{q} \notin\{0\}_{P}$, then $a \neq 0$, 
that is $a \notin\{0\}$. Now, $\frac{r a}{p q} \in\{0\}_{P}$ implies that $\operatorname{tra}=0 \in\{0\}$, for some $t \notin P$. Since, $\{0\}$ is a prime submodule of $M$, then we get $\operatorname{tr} M=\{0\}$, then we get $(\operatorname{trM})_{P}=\{0\}_{P}$, and as $(t r M)_{P}=\frac{t r}{t p} M_{P}$, we get $\frac{t r}{t p} M_{P}=\{0\}_{P}$, then we get ${ }_{p}^{r} M_{P}=\frac{t}{t} \frac{r}{p} M_{P}=\frac{t r}{t p} M_{P}=\{0\}_{P}$. Hence, $\{0\}_{P}$ is a prime submodule of $M_{P}$, so that $M_{P}$ is a prime module that means $M$ is a locally prime module.

The following result shows that under certain conditions the localization of a prime submodule is prime.

Theorem 2.4. Let $M$ be a locally Artinian $R$ - module. If $P$ is a maximal ideal of $R$ and $N$ is a primal submodule of $M$ with $S_{M}(N) \subseteq P$ and $(N: M)$ is a maximal ideal of $R$, then $N_{P}$ is a prime submodule of $M$.

Proof. As $N$ is primal and $(N: M)$ is a maximal ideal of $R$, by [8, Proposition 2.24], we get $N$ is a prime submodule of $M$ and as $S_{M}(N) \subseteq P$, so by [8, Proposition 2.21], we get $N_{P}$ is a prime submodule of $M$.

Theorem 2.5. Let $M$ be an $R$ - module, $N$ a proper submodule of $M$ with $S_{M}(N) \subseteq P$ and $P$ a maximal ideal of $R$. If $N_{P}$ is a prime submodule of $M_{P}$, then $(N: M)$ is a maximal ideal of $R$.

Proof. Since, $N_{P}$ is a prime submodule of $M_{P}$ and $S_{M}(N) \subseteq P$, so by [8, Proposition 2.21], we get $N$ is a prime submodule of $M$ and then by [8, Proposition 2.23], we get $(N: M)$ is a maximal ideal of $R$.

Theorem 2.6. Let $M$ be an Artinian $R$-module and $P$ a maximal ideal of $R$. If $N$ is a proper submodule of $M$ such that $S_{M}(N) \subseteq P$, then $N_{P}$ is a prime submodule of $M_{P}$ if and only if (N:M) is a maximal ideal of $R$.

Proof. $\Leftrightarrow)$ Let $N_{P}$ be a prime submodule of $M_{P}$. Since we have, $S_{M}(N) \subseteq P$, so by [8, Proposition 2.21], we get $N$ is a prime submodule and then by [3, Corollary 2.4], we get $(N: M)$ is a maximal ideal of $R$.

$(\Leftrightarrow)$ Let $(N: M)$ be a maximal ideal of $R$, then by [3, Corollary 2.4], we get $N$ is a prime submodule and as $S_{M}(N) \subseteq P$, by [8, Proposition 2.21], we get $N_{P}$ is a prime submodule of $M_{P}$.

In the following result we give some conditions which make the localization of a locally Artinian module as a prime module.

Theorem 2.7. Let $M$ be a locally Artinian $R$-module and $P$ a maximal ideal of $R$ such that $S_{M}(0) \subseteq J(R)$. If $A n n(M)$ is a primal ideal of $R$, then $M_{P}$ is a prime $R_{P}$ - module if and only if $R /(\operatorname{Ann}(M))$ is a field.

Proof. $\Leftrightarrow$ Let $M_{P}$ be a prime module. Now, we have $S_{M}(0) \subseteq J(R) \subseteq P$, thus by [8, Proposition 2.14], we get $M$ is a prime module and by [8, Proposition 2.17], we have $R /(\operatorname{Ann}(M))$ is a field. 
$(\Leftarrow)$ As $S_{M}(0) \subseteq J(R)$, we have $\operatorname{Ann}(M)$ is a primal ideal of $R$ and $R /(\operatorname{Ann}(M))$ is a field and by [8, Proposition 2.17], we get $M$ is a prime module, then by [9, Theorem 2.11], we get $M_{P}$ is a prime $R_{P}$-module.

The next result proves that under certain condition the localization of a locally prime module is a prime module.

Theorem 2.8. Let $M$ be an $R$ - module. If $M$ is locally prime and $S_{M}(0) \subseteq J(R)$, then $M_{P}$ is a prime module.

Proof. By [8, Corollary 2.15], we get $M$ is a prime module and by [9, Theorem 2.11], we have $M_{P}$ is prime.

In the following result we give some conditions under which we can characterize those faithful locally Artinian modules the localization of which are prime.

Theorem 2.9. Let $M$ be a faithful localy Artinian $R-$ module and $P$ be a maximal ideal of $R$. If $R$ is a primal ring and $S_{M}(0) \subseteq J(R)$, then $M_{P}$ is a prime module if and only if $R$ is a field.

Proof $(\Rightarrow)$ Let $M_{P}$ be a prime and $P$ be a maximal ideal of $R$, then $S_{M}(0) \subseteq J(R) \subseteq P$, thus by [8, Proposition 2.14], we get $M$ is prime and by [8, Corollary 2.19], we have $R$ is a field.

$(\Leftarrow)$ By [8, Corollary 2.19], we get $M$ is prime and by [9, Theorem 2.11], we have $M_{P}$ is a prime.

Theorem 2.10. Let $M$ be an $R$ - module and $N$ be a proper submodule of $M$. If $P$ is a maximal ideal of $R$ such that $S_{M}(N) \subseteq P$ and the DCC is satisfied on cyclic submodules of $M_{P}$, then $N_{P}$ is a prime submodule of $M_{P}$ if and only if $N_{P}$ is a weakly prime submodule of $M_{P}$.

Proof. $(\Rightarrow)$ Let $N_{P}$ be a prime submodule of $M_{P}$, then by [8, Proposition 2.21], we get $N$ is a prime submodule of $M$, so by [8, Corollary 2.22], we get $N$ is a weakly prime submodule of $M$ and by [8, Proposition 2.21], we have $N_{P}$ is a weakly prime submodule of $M_{P}$.

$(\Leftarrow)$ Let $N_{P}$ be a weakly prime submodule of $M_{P}$, then by [8, Proposition 2.21], we get $N$ is a weakly prime submodule of $M$, so by [8, Corollary 2.22], we get $N$ is a prime submodule of $M$ and by [8, Proposition 2.21], we have $N_{P}$ is a prime submodule of $M_{P}$.

In the following two results we give some further conditions under which the localization of (faithful) Artinian modules are prime.

Theorem 2.11. Let $M$ ba an Artinian $R$ - module and $P$ ba a maximal ideal of $R$ such that $S_{M}(0) \subseteq P$, then $M_{P}$ is a prime module if and only if $R /(\operatorname{Ann}(M))$ is a field.

Proof $(\Rightarrow)$ Let $M_{P}$ be a prime module and $S_{M}(0) \subseteq P$, then by [8, Proposition 2.14], we get $M$ is a prime module and by [3, Proposition 2.1], we have $R /(\operatorname{Ann}(M))$ is a field. 
$(\Leftarrow)$ By [3, Proposition 2.1], we have $M$ is a prime module and by [9, Theorem 2.11 , we get $M_{P}$ is a prime module.

Theorem 2.12. Let $M$ be a faithful Artinian $R$ - module and $P$ ba a maximal ideal of $R$ such that $S_{M}(0) \subseteq P$, then $M_{P}$ is a prime module if and only if $R$ is a field.

Proof. $(\Rightarrow)$ Let $M_{P}$ be a prime module. As, $S_{M}(0) \subseteq P$, by [8, Proposition 2.14], we get $M$ is a prime module and by [3, Corollary 2.2], we have $R$ is a field.

$(\Leftarrow)$ Let $R$ be a field. By [3, Corollary 2.2], we have $M$ is a prime module and by [9, Theorem 2.11], we get $M_{P}$ is a prime module.

\section{Strongly Reduced, Primally Reduced and Radically Reduced Modules}

In this section, further types of modules are studied and investigated such as, strongly reduced modules, primally reduced modules, radically reduced modules and some other types. Some properties of these types of modules are proved and some relations between them are determined and also some characterizations of them are given.

In the first result we prove that under certain condition, if the localization of a module is reduced, then the module itself is also reduced.

Theorem 3.1. Let $M$ be an $R$-module and $P$ be a maximal ideal of $R$ such that $S_{M}(0) \subseteq P$. If $M_{P}$ is a reduced $R_{P}$-module, then $M$ is a reduced $R$-module.

Proof. Let $x \in \cap \operatorname{Spec}(M)$. Let $\bar{N} \in \operatorname{Spec}\left(M_{P}\right)$, so that $\bar{N}$ is prime submodule of $M_{P}$. Then by [8, Lemma 2.27], we have $\bar{N}=N_{P}$ for the prime submodule $N=\left\{x \in M: \frac{x}{1} \in \bar{N}\right\}$ of $M$ with $S_{M}(N) \subseteq P$, that means $N_{P}$ is a prime submodule of $M_{P}$, then by [8, Lemma 2.27], we get $N$ is a prime submodule of $M$, that means $N \in \operatorname{Spec}(M)$, so we get $x \in N$ and then $\frac{x}{1} \in N_{P}=\bar{N}$, thus we get $\frac{x}{1} \in \cap \operatorname{Spec}\left(M_{P}\right)$, but $\cap \operatorname{Spec}\left(M_{P}\right)=0$ we get $\frac{x}{1}=0$ and as $S_{M}(0) \subseteq P$, by $[8$, Lemma 2.1], we get $x=0$, so we have $\cap \operatorname{Spec}(M)=0$. That means $M$ is a reduced module.

The next result shows that the localization of strongly reduced modules are also strongly reduced.

Theorem 3.2. Let $M$ be an $R$-module and $P$ be a maximal ideal of $R$. If $M$ is strongly reduced, then $M_{P}$ is strongly reduced.

Proof. Let $\frac{x}{p} \in \cap \operatorname{Spec}_{P}\left(M_{P}\right)$, where $x \in M$ and $p \notin P$. Let $N \in \cap \operatorname{Spec}_{P}(M)$, then $N \in \operatorname{Spec}(M)$ and $S_{M}(N) \subseteq P$, so by [8, Proposition 2.20], we get $S_{M_{P}}\left(N_{P}\right)=\left(S_{M}(N)\right)_{P} \subseteq P_{P}$, that means $S_{M_{P}}\left(N_{P}\right) \subseteq P_{P}$ and then by [8, Proposition 2.21], we get $N_{P}$ is a prime submodule of $M_{P}$, that means $N_{P} \in \operatorname{Spec}\left(M_{P}\right)$ and $S_{M_{P}}\left(N_{P}\right) \subseteq P_{P}$, we get $N_{P} \in \operatorname{Spec}_{P}\left(M_{P}\right)$, so that $\frac{x}{p} \in N_{P}$ and by [8, Lemma 2.1], we have $x \in N$, thus we get $x \in \cap \operatorname{Spec}_{P}(M)$, but $\cap \operatorname{Spec}_{P}(M)=0$ we get $x=0$ and 
$p \notin P$ then $\frac{x}{p}=\frac{0}{p}=0$, so we have $\cap \operatorname{Spec}_{P}\left(M_{P}\right)=0$, that means $M_{P}$ is strongly reduced.

In the following result we give a condition under which the converse of the last theorem is true.

Theorem 3.3. Let $M$ be an $R$-module and $P$ be a prime ideal of $R$ such that $S_{M}(0) \subseteq P$. If $M_{P}$ is a strongly reduced $R_{P}$-module, then $M$ is a strongly reduced module.

Proof. Let $x \in \cap \operatorname{Spec}_{P}(M)$ and let $\bar{N} \in \operatorname{Spec}_{P}\left(M_{P}\right)$, that is $\bar{N}$ is prime submodule of $M_{P}$ and $S_{M_{P}}(\bar{N}) \subseteq P_{P}$. Then by [8, Lemma 2.27], we have $\bar{N}=N_{P}$ for the prime submodule $N=\left\{x \in M: \frac{x}{1} \in \bar{N}\right\}$ of $M$ with $S_{M}(N) \subseteq P$, that means $N_{P}$ is a prime submodule of $M_{P}$ and $S_{M_{P}}\left(N_{P}\right) \subseteq P_{P}$, that means $N \in \operatorname{Spec}_{P}(M)$, so we get $x \in N$ and $p \notin P$, then we have $\frac{x}{p} \in N_{P}=\bar{N}$, thus we get $\frac{x}{p} \in \cap \operatorname{Spec}_{P}(\bar{N})$ and as $\cap \operatorname{Spec}_{P}(\bar{N})=0$, we get $\frac{x}{p}=0$, then by [8, Lemma 2.1], we get $x=0$, thus we have $\cap \operatorname{Spec}_{P}(M)=0$. That means, $M$ is strongly reduced.

Next we prove that, under a certain condition those modules localization of which are strongly reduced are reduced.

Corollary 3.4. If $M_{P}$ is strongly reduced and $P$ be a maximal ideal of $R$ such that $S_{M}(0) \subseteq P$, then $M$ is reduced.

Proof. Since, $M_{P}$ is a strongly reduced $R_{P}$-module, so by Theorem 3.3, we get $M$ is a strongly reduced $R$-module, that gives $\cap \operatorname{Spec}_{P}(M)=0$. Then by $[6$, Theorem 2.4], we get $\cap \operatorname{Spec}(M)=0$.

Theorem 3.5. Let $M$ be an $R-$ module and $P$ a maximal ideal of $R$, then we have $\left(\cap \operatorname{Spec}_{P}(M)\right)_{P}=\cap \operatorname{Spec}_{P}\left(M_{P}\right)$.

Proof. Let $\frac{x}{p} \in\left(\cap \operatorname{Spec}_{P}(M)\right)_{P}$, for $x \in M$ and $p \notin P$. Then $q x \in \cap \operatorname{Spec}_{P}(M)$, for some $q \notin P$. Let $\bar{N} \in \operatorname{Spec}_{P}\left(M_{P}\right)$, that is $\bar{N}$ is prime submodule of $M_{P}$. Then by [8, Lemma 2.27], we have $\bar{N}=N_{P}$ for the prime submodule $N=\left\{x \in M: \frac{x}{1} \in \bar{N}\right\}$ of $M$ with $S_{M}(N) \subseteq P$, so that $N \in \operatorname{Spec}_{P}(M)$ and thus $q x \in N$, from which we get $\frac{x}{p}=\frac{q}{q} \frac{x}{p}=\frac{q x}{q p} \in N_{P}=\bar{N}$ and so $\frac{x}{p} \in \cap \operatorname{Spec}_{P}\left(M_{P}\right)$ and thus we have $\left(\cap \operatorname{Spec}_{P}(M)\right)_{P} \subseteq \cap \operatorname{Spec}_{P}\left(M_{P}\right)$. Now, let $\frac{x}{p} \in \cap \operatorname{Spec}_{P}\left(M_{P}\right)$, where $x \in M$ and $p \notin P$. Let $N \in \operatorname{Spec}_{P}(M)$, so that $N \in \operatorname{Spec}(M)$ and $S_{M}(N) \subseteq P$, then by [8, Proposition 2.20], we get $S_{M_{P}}\left(N_{P}\right)=\left(S_{M}(N)\right)_{P} \subseteq P_{P}$, that means $S_{M_{P}}\left(N_{P}\right) \subseteq P_{P}$ and by [8, Proposition 2.21], we get $N_{P}$ is a prime submodule of $M_{P}$, that is $N_{P} \in \operatorname{Spec}_{P}\left(M_{P}\right)$, so that $\frac{x}{p} \in N_{P}$ and by [8, Lemma 2.1], we have $x \in N$, thus we get $x \in \cap \operatorname{Spec}_{P}(M)$, this gives $\frac{x}{p} \in\left(\cap \operatorname{Spec}_{P}(M)\right)_{P}$, so that $\cap \operatorname{Spec}_{P}\left(M_{P}\right) \subseteq\left(\cap \operatorname{Spec}_{P}(M)\right)_{P}$. Hence, we get $\left(\cap \operatorname{Spec}_{P}(M)\right)_{P}=\cap \operatorname{Spec}_{P}\left(M_{P}\right)$. 
The following corollary proves that, if the localization of a module is strongly reduced, then this localization is reduced.

Corollary 3.6. Let $M$ be an $R$-module and $P$ a maximal ideal of $R$. If $M_{P}$ is a strongly reduced $R_{P}$-module, then $M_{P}$ is a reduced $R_{P}-$ module.

Proof. Since, $M_{P}$ is strongly reduced so that $\cap \operatorname{Spec}_{P}\left(M_{P}\right)=0$. Then by Theorem 3.5, we get $\left(\cap \operatorname{Spec}_{P}(M)\right)_{P}=0$ and by [6, Theorem 2.4], we get $\cap \operatorname{Spec}\left(M_{P}\right)=0$, that means, $M_{P}$ is reduced.

Next, we prove that the localization of strongly reduced modules are reduced.

Corollary 3.7. Let $M$ be an $R$-module and $P$ a maximal ideal of $R$. If $M$ is a strongly reduced $R$-module, then $M_{P}$ is a reduced $R_{P}$-module.

Proof. Since, $M$ is strongly reduced, so by Theorem 3.2, we get $M_{P}$ is a strongly reduced $R_{P}$ - module that is $\cap \operatorname{Spec}_{P}\left(M_{P}\right)=0$. By Theorem 3.5, we have $\left(\cap \operatorname{Spec}_{P}(M)\right)_{P}=\cap \operatorname{Spec}_{P}\left(M_{P}\right)=0$ and then by [6, Theorem 2.4], we get $\cap \operatorname{Spec}\left(M_{P}\right)=0$.

In the following theorem, we prove that the localization of radically reduced modules are radically reduced.

Theorem 3.8. Let $M$ be an $R$-module and $P$ be a maximal ideal of $R$. If $M$ is radically reduced, then $M_{P}$ is radically reduced.

Proof. Let $\frac{x}{p} \in \cap \operatorname{jpSpec}\left(M_{P}\right)$, where $x \in M$ and $p \notin P$. Let $N \in \cap j p \operatorname{Spec}(M)$, then $N \in p \operatorname{Spec}(M)$ and $S_{M}(N) \subseteq \operatorname{Rad}_{j}(R) \subseteq P$. By [5, Proposition 2.5], $N_{P}$ is a primal submodule of $M_{P}$, that is $N_{P} \in p \operatorname{Spec}\left(M_{P}\right)$, so that $S_{M_{P}}\left(N_{P}\right)$ is a proper ideal of $R_{P}$ and since, $R_{P}$ is a local ring with the unique maximal ideal $P_{P}$, so that $S_{M_{P}}\left(N_{P}\right) \subseteq P_{P}=\operatorname{Rad}_{j}\left(R_{P}\right)$, so that $\frac{x}{p} \in N_{P}$ and then by [8, Lemma 2.1], we have $x \in N$, thus we get $x \in \cap j p \operatorname{Spec}(M)$, but $\cap j p \operatorname{Spec}(M)=0$ we get $x=0$ and $p \notin P$ then $\frac{x}{p}=\frac{0}{p}=0$, we have $\cap \operatorname{jpSpec}\left(M_{P}\right)=0$ that means $M_{P}$ is radically reduced.

Now, for the local rings we give a condition which makes the converse of the theorem is also true.

Theorem 3.9. Let $R$ be a local ring with $P$ as its unique maximal ideal and $M$ be an $R$-module such that $S_{M}(0) \subseteq P$. If $M_{P}$ is radically reduced, then $M$ is radically reduced.

Proof. Let $x \in \cap \operatorname{jpSpec}(M)$ and $\bar{N} \in \operatorname{jpSpec}\left(M_{P}\right)$, that is $\bar{N}$ is primal submodule of $M_{P}$. Then by [5, Proposition 2.6], we have $\bar{N}=N_{P}$ for the primal submodule $N=\left\{x \in M: \frac{x}{1} \in \bar{N}\right\}$ of $M$ with $S_{M}(N) \subseteq P$, that means $N_{P}$ is a primal submodule of $M_{P}$ and $S_{M_{P}}\left(N_{P}\right) \subseteq \operatorname{Rad}_{j}\left(R_{P}\right)=P_{P}$ and by [5, Proposition 2.6], we get $N$ is a primal submodule of $M$, so that $N \in \operatorname{pSpec}(M)$ and $S_{M}(N)$ is a (proper) ideal of $R$ and thus we get $S_{M}(N) \subseteq P\left(=\operatorname{Rad}_{j}(R)\right)$, which means that $N \in j p \operatorname{Spec}(M)$, then 
$x \in N$ and $p \notin P$, we have $\frac{x}{p} \in N_{P}$, thus we get $\frac{x}{p} \in \cap \operatorname{jpSpec}\left(N_{P}\right)$, but กjpSpec $\left(N_{P}\right)=0$ we get $\frac{x}{p}=0$, so by [8, Lemma 2.1], we get $x=0$, then we have $\cap j p \operatorname{Spec}(M)=0$. That means $M$ is a radically reduced $R$-module.

Next, we determine a relation between the primal spectrum and the Jacobson radical of a module.

Theorem 3.10. Let $M$ be an $R$ - module and let $P$ be a maximal ideal of $R$, then $\cap p \operatorname{Spec}(M) \subseteq \cap j p \operatorname{Spec}(M)$.

Proof. As, $j p \operatorname{Spec}(M) \subseteq p \operatorname{Spec}(M)$, we get $\cap p \operatorname{Spec}(M) \subseteq \cap j p S p e c(M)$.

The following theorem shows that the localization of the Jacobson radical of a module and the Jacobson radical of the localization are the same.

Theorem 3.11. Let $R$ be a local ring with $P$ as its unique maximal ideal and $M$ be an $R$-module, then we have $(\cap j p S p e c(M))_{P}=\cap j p S p e c\left(M_{P}\right)$.

Proof. Let $\frac{x}{p} \in(\cap j p \operatorname{Spec}(M))_{P}$, where $x \in M$ and $p \notin P$. Then, there exists $q \notin P$ such that $q x \in \cap j p \operatorname{Spec}(M)$. Now, let $\bar{N} \in j p \operatorname{Spec}\left(M_{P}\right)$, that is $\bar{N}$ is primal submodule of $M_{P}$. Then by [5, Proposition 2.6], we have $\bar{N}=N_{P}$ for the primal submodule $N=\left\{x \in M: \frac{x}{1} \in \bar{N}\right\}$ of $M$ with $S_{M}(N) \subseteq P$, that is $N_{P} \in p \operatorname{Spec}\left(M_{P}\right)$ and $S_{M_{P}}\left(N_{P}\right) \subseteq \operatorname{Rad}_{j}\left(R_{P}\right)=P_{P}$ and by [5, Proposition 2.6], we get $N$ is a primal submodule of $M$, so that $N \in p \operatorname{Spec}(M)$ and $S_{M}(N)$ is a (proper) ideal of $R$ and thus we get $S_{M}(N) \subseteq P\left(=\operatorname{Rad}_{j}(R)\right)$, which means that $N \in j p S p e c(M)$ and thus $q x \in N$, from which we get $\frac{x}{p}=\frac{q}{q} \frac{x}{p}=\frac{q x}{q p} \in N_{P}=\bar{N}$, so that $\frac{x}{p} \in \operatorname{njpSpec}\left(M_{P}\right)$ and thus we have $(\cap j p \operatorname{Spec}(M))_{P} \subseteq \cap \operatorname{jpSpec}\left(M_{P}\right)$. Now, let $\frac{x}{p} \in \cap \operatorname{jpSpec}\left(M_{P}\right)$, where $x \in M$ and $p \notin P$. Let $N \in j p \operatorname{Spec}(M)$, then $N \in p \operatorname{Spec}(M)$ and $S_{M}(N) \subseteq \operatorname{Rad}_{j}(R) \subseteq P$. By [5, Proposition 2.5], $N_{P}$ is a primal submodule of $M_{P}$, that is $N_{P} \in \operatorname{pSpec}\left(M_{P}\right)$, so that $S_{M_{P}}\left(N_{P}\right)$ is a proper ideal of $R_{P}$ and since, $R_{P}$ is a local ring with the unique maximal ideal $P_{P}$, so that $S_{M_{P}}\left(N_{P}\right) \subseteq P_{P}=\operatorname{Rad}_{j}\left(R_{P}\right)$, we get $N_{P} \in j p \operatorname{Spec}\left(M_{P}\right)$, so that $\frac{x}{p} \in N_{P}$ and then by [8, Lemma 2.1], we have $x \in N$, thus we get $x \in \cap \operatorname{jpSpec}(M)$, this gives $\frac{x}{p} \in(\cap j p \operatorname{Spec}(M))_{P}$, so that $\cap j p S p e c\left(M_{P}\right) \subseteq(\cap j p S p e c(M))_{P}$. Hence, we get $(\cap j p S p e c(M))_{P}=\cap j p S p e c\left(M_{P}\right)$.

The following corollary shows that, radically reducedness property implies primally reducedness for the localized module.

Corollary 3.12. Let $R$ be a local ring with $P$ as its unique maximal ideal and $M$ be an $R$-module. If $M_{P}$ is radically reduced, then $M_{P}$ is primally reduced.

Proof. Since, $M_{P}$ is a radically reduced, so that $\cap j p \operatorname{Spec}\left(M_{P}\right)=0$. Then, by Theorem 3.11, we get $(\operatorname{NpSpec}(M))_{P}=0$ and by [5, Theorem 2.11], we get 
$\cap p \operatorname{Spec}\left(M_{P}\right) \subseteq(\cap \operatorname{jpSpec}(M))_{P}=0$ that means $M_{P}$ is a primally reduced $R_{P}$-module.

In the following corollary, we give a condition which makes those modules the localization of which are radically reduced are primally reduced.

Corollary 3.13. Let $R$ be a local ring with $P$ as its unique maximal ideal and $M$ be an $R$-module such that $S_{M}(0) \subseteq P$. If $M_{P}$ is radically reduced, then $M$ is primally reduced.

Proof. By Theorem 3.9, we have $M$ is a radically reduced and by [5, Corollary 2.12], we get $M$ is a primally reduced $R$-module.

Corollary 3.14. Let $M$ be a multiplication and a locally reduced $R$ - module and $N$ be a submodule of $M$. If $M_{P}$ is a primally reduced $R$ - module, then $N \cap \operatorname{Ann}(N) M=0$.

Proof. By [5, Corollary 2.9], we have $M$ is primally reduced and by [5, Proposition 2.17], we get $N \cap \operatorname{Ann}(N) M=0$.

In the following theorem, we give a condition which makes reduced modules, radically reduced modules and primally reduced modules equivalent.

Theorem 3.15. Let $R$ be a local ring with $P$ as its uniqe maximal ideal and $M$ be an $R$-module such that $S_{M}(0) \subseteq P$. The following statements are equivalent:

(1) $M_{P}$ is radically reduced.

(2) $M$ is primally reduced.

(3) $M$ is reduced.

Proof. $(1 \Rightarrow 2)$ By Corollary 3.13, we have $M$ is a primally reduced $R$-module. $(2 \Rightarrow 3)$ By [5, Theorem 2.16], we get $M$ is a reduced $R$-module.

$(3 \Rightarrow 1)$ By [5, Theorem 2.16], we get $M$ is radically reduced and by Theorem 3.8, we get $M_{P}$ is radically reduced.

By assuming some conditions in the following theorem, we give a necessary and sufficient condition for a submodule to have a weakly prime localization.

Theorem 3.16. Let $M$ be an $R$-module and $N$ a proper submodule of $M$ with $S_{M}(0) \subseteq(N: M)$. If $P$ is a maximal ideal of $R$ such that $S_{M}(N) \subseteq P$, then $N_{P}$ is weakly prime if and only if $S_{M}(N) \subseteq(N: M)$.

Proof. $\left(\Rightarrow\right.$ ) Let $N_{P}$ be weakly prime, then by [8, Proposition 2.21], we get $N$ is weakly prime and then by [5, Proposition 2.15], we get $S_{M}(N) \subseteq(N: M)$.

$\Leftrightarrow)$ suppose that $S_{M}(N) \subseteq(N: M)$. By [5, Proposition 2.15], we get $N$ is weakly prime and by [8, Proposition 2.21], we get $N_{p}$ is weakly prime.

Next, we give some conditions which make the modules that have weak multiplication localization as weak multiplication modules.

Theorem 3.17. Let $M$ be an $R$-module and $N$ a proper submodule of $M$ with $S_{M}(N) \subseteq J(R)$. If $P$ is a maximal ideal of $R$ such that $M_{P}$ is a weak multiplication $R_{P}$-module, then $M$ is a weak multiplication $R$-module. 
Proof. Let $P$ be any maximal ideal of $R$, then $S_{M}(N) \subseteq J(R) \subseteq P$. If $N$ ia any prime submodule of $M$, then by [8, Proposition 2.21], we get $N_{P}$ is a prime submodule of $M_{P}$ and as $M_{P}$ is a weak multiplication module, we have $N_{P}=\left(N_{P}: M_{P}\right) M_{P}$, then by [7, Theorem 2.21], we get $N_{P}=((N: M) M)_{P}$, so by [7, Corollary 2.2], we get $N=(N: M) M$, that means $M$ is a weak multiplication $R-$ module.

In the following theorem, we prove that the localization of prime and regular modules are fully prime.

Theorem 3.18. Let $M$ be an $R$-module and $P$ be a prime ideal of $R$. If $M$ is a prime and regular module, then $M_{P}$ is a fully prime $R_{P}$-module.

Proof. By [4, Corollary 1.9], we get $M$ is a fully prime module and by [9, Theorem 2.1 , we get $M_{P}$ is a fully prime $R_{P}$-module.

Theorem 3.19. If each cyclic submodule of an $R$-module $M$ is a prime submodule and $P$ is a prime ideal of $R$, then $M_{P}$ is prime and each cyclic submodule of $M$ is semiprime.

Proof. By [4, Corollary 1.9], we get $M$ is prime and each cyclic submodule of $M$ is semiprime and by [9, Theorem 2.11], we get $M_{P}$ is prime.

Theorem 3.20. Let $M$ be an $R$-module and $N$ be a proper submodule of $M$. If $P$ is a maximal ideal of $R$ with $S_{M}(N) \subseteq P$ and $(N: M)$ is a maximal ideal of $R$, then $(N: M)_{P}$ is a maximal ideal of $R_{P}$.

Proof. Since $1 \notin P$, implies that $\frac{1}{1}$ is the identity of $R_{P}$ and $(N: M)_{P}=R_{P}$, let $\frac{1}{1} \in(N: M)_{P}=\left(N_{P}: M_{P}\right)$, implies that $\frac{1}{1} M_{P} \subseteq N_{P}$, we get $M_{P} \subseteq N_{P}$, that means $M_{P}=N_{P}$, which is a contradiction. To show that $(N: M)_{P}$ is a maximal ideal of $R_{P}$, so let $(N: M)_{P} \subseteq \bar{J} \subseteq R_{P}$, for the ideal $\bar{J}$ of $R_{P}$. By [7, Proposition 2.16], we have $\bar{J}=J_{P}$, for the ideal $J=\left\{a \in R: \frac{a}{1} \in \bar{J}\right\}$ of $R$, so that $(N: M)_{P} \subseteq J_{P} \subseteq R_{P}$. Suppose that $J_{P} \neq R_{P}$, so that $J \neq R$. If $a \in(N: M)$, then $\frac{a}{1} \in J_{P}$, so $p a \in J$, for some $p \notin P$, then $\frac{p a}{1} \in \bar{J}$. Now, $\frac{a}{1}=\frac{1}{p} \frac{p a}{1} \in \bar{J}$, so that $a \in J$ and thus $(N: M) \subseteq J \subseteq R$. As $(N: M)$ is a maximal, we get $(N: M)=J$, so that $(N: M)_{P}=J_{P}$, that means $(N: M)_{P}$ is a maximal ideal of $R_{P}$.

Theorem 3.21. Let $M$ be a locally Artinian $R$-module and $N$ a proper submodule of $M$. If $P$ is a maximal ideal of $R$ with $S_{M}(N) \subseteq P$ and $(N: M)_{P}$ is a maximal ideal of $R_{P}$, then $(N: M)$ is a maximal ideal of $R$.

Proof. As $N$ is proper, by [7, Proposition 2.17], we get $N_{P}$ is a proper submodule of $M_{P}$ and then by [7, Theorem 2.21], we have $(N: M)_{P}=\left(N_{P}: M_{P}\right)$ and Since, $(N: M)_{P}$ is a maximal ideal of $R_{P}$ and $M_{P}$ is an Artinian module, by [3, Corollary 2.4], we have $N_{P}$ is a prime submodule of $M_{P}$ and by [8, Proposition 2.21], we get $N$ is a prime submodule of $M$ and by [8, Proposition 2.23], we get $(N: M)$ is a maximal ideal of $R$. 


\section{References:}

[1] Atani, S. E. and Darani, A. Y. : Notes on the Primal Submodules, Chiang Mai J. Sci.

35(3), 2008, pp 399-410.

[2] Atani, S. E. and Farzalipour, F. : On Weakly Prime Submodules, Tamkang Journal of Mathematics, Vol. 38, No. 3, 2007, 247-252.

[3] Azizi, A.: Prime Submodules of Artinian Modules, Taiwaness Journal of Mathematics, Vol. 13, No. 6B, pp. 2011-2020, 2009.

[4] Behboodi, M. , Karamzadeh, O.A.S. and Koohy, H. : Modules Whose Certain Submodules Are Prime, Vietnam Journal of Mathematics 32:3 (2004) pp 303-307.

[5] Jabbar, A. K.: A Generalization of Reduced Modules, International Journal of Algebra, Vol. 8, 2014, no. 1, 39-45.

[6] Jabbar, A. K.: On Locally Reduced and Locally Multiplication Modules, International Mathematical Forum, Vol. 8, 2013, no. 18, 851-858,

[7] Jabbar, A. K.: A Generalization of prime and weakly prime submodules, Pure Mathematical Sciences, Vol. 2, 2013, No. 1, 1-11,

[8] Jabbar, A. K.: On Locally Artinian Modules, International Journal of Algebra, Vol. 6, 2012, No. 27, 1325-1334.

[9] Jabbar, A. K. and Mohammad R. R.: The Effect of Localization on Properties of Certain Types of Modules, The $2^{\text {nd }}$ International conference of the college of Education - University of Garmian held on 21-22/8/2016.

[10] Larsen, M. D. and McCarthy, P. J.: Multiplicative Theory of Ideals, Academic Press, New York and London, 1971.

[11] Lomp, C. and Pena, P. A. J. : A Note on Prime Modules, Divulgaciones Mathematicas, Vol. 8, No. 1, 2000, pp 31-42.

[12] Rajaee, S. : Comaximal Submodules of Multiplication Modules, International Mathematical Forum, Vol.5, 2010, no. 24, 1179-1183. 